\title{
Structure of the fibrin protofibril
}

\author{
(electron microscopy/self-assembly/polymerization)
}

\author{
W. E. Fowler ${ }^{* \dagger} \dagger$ R. R. Hantgan $\ddagger$, J. Hermans ${ }^{\ddagger}$, AND H. P. ERICKSON ${ }^{* \S}$ \\ *Department of Anatomy, Duke University Medical Center, Durham, North Carolina 27710; and ¥Department of Biochemistry, University of North Carolina, Chapel \\ Hill, North Carolina 27514
}

Communicated by Robert L. Hill, May 7, 1981

\begin{abstract}
We identified the two-stranded fibrin protofibril and studied its structure in electron micrographs of negatively stained specimens. Based on these images and on considerations of symmetry, we constructed a model of the protofibril in which the two strands of trinodular fibrin molecules are related by a twofold screw axis between the strands and two-fold axes perpendicular to them. The two strands are held together by staggered lateral contacts between the central nodules of one strand and outer nodules of the other. The molecules within a strand are joined by longitudinal contacts between outer nodules. This interpretation of the structure of protofibrils is supported by images of trimer complexes whose preparation and structure are described here, in which the central nodule of a fibrin monomer is attached to the crosslinked outer nodules of two other molecules. We conclude that the association of protofibrils to form thicker fibers must involve a second type of lateral contact, probably between outer nodules of adjacent, in-register strands. In total, we identify three intermolecular contacts involved in the polymerization of fibrin.
\end{abstract}

The plasma protein fibrinogen, the major structural component of the blood clot, is an elongated molecule whose shape is a trinodular rod, $45 \mathrm{~nm}$ long $(1,2)$. Evidence has been presented recently for a finer subdivision into seven nodules (3), but the description of two 7-nm-diameter outer nodules connected by thin linking rods to a 5-nm-diameter central nodule is sufficient for the present work. Fibrinogen is composed of two identical half molecules, which are probably related by an exact or approximate two-fold axis through the central nodule $(4,5)$. The two-fold molecular symmetry is consistent with the overall shape of the molecule, with the identification of the nodules with specific parts of the sequence $(6,7)$, and with the measurements of periodicities in fibrin and crystalline aggregates $(3,4,8)$. The central nodule is the site of activation by the protease thrombin, which cleaves off the small fibrinopeptides to form fibrin monomer. The fibrin monomer is essentially identical in structure to fibrinogen, but instead of being soluble, it spontaneously polymerizes to form fibrin fibers.

The most informative structural feature seen in electron micrographs of fibrin fibers is a pattern of transverse bands, which repeat every $22.5 \mathrm{~nm}$ or exactly one-half the length of the molecule. The generally accepted interpretation of this banding pattern is that the rod-like molecules are parallel to the fiber axis; the molecules are arranged end-to-end to form strands, which are one molecule thick; and alternate strands are staggered by one-half the length of the molecule (8).

Ferry identified a two-stranded polymer of fibrin, which he called a protofibril, and proposed a two-step mechanism for the polymerization of fibrin (9). In the first step, fibrin monomers polymerize to form protofibrils; in the second step, these protofibrils associate laterally to form the thicker fibrin fibers. Re-

The publication costs of this article were defrayed in part by page charge payment. This article must therefore be hereby marked "advertisement" in accordance with $18 \mathrm{U}$. S. C. $\$ 1734$ solely to indicate this fact. cent evidence from light scattering $(10)$ confirmed this two-step mechanism by showing that a two-stranded protofibril was an early polymer and, presumably, an intermediate in the normal polymerization of fibrin.

Protofibrils also have been identified by electron microscopy $(11,12)$, but the structural interpretation was limited by the resolution of the shadowed specimens used in these studies. Nevertheless, the long, thin fibrils had the thickness expected for a two-stranded protofibril, and there were definite repeating features, which Krakow et al. (11) interpreted as showing a staggered arrangement of trinodular molecules.

Negative staining is often preferable to shadowing because it provides higher resolution and better definition of details in complicated structures. Using the negative-stain technique that allowed us to visualize the trinodular structure of the fibrinogen molecule (2), we have identified protofibrils in the early stages of fibrin polymerization and correlated their presence with data from a parallel study by light scattering (13). In that study, we also found the progression from two-stranded protofibrils to larger polymers was unchanged by the action of factor XIIIa. Because the small polymers were crosslinked as they were formed in this reaction, we concluded that the two-stranded protofibrils were incorporated intact into the larger polymers and, thus, were a true intermediate in the polymerization. In this paper we give our analysis of the images of negatively stained protofibrils, which confirms and extends the interpretation of Krakow et al. (11) regarding the structure of the protofibril. In addition, we present images of a related trimer complex, which demonstrate more clearly the validity of this interpretation.

Several different models for fibrin polymerization have been proposed recently, all incorporating a staggered arrangement of trinodular molecules but with different symmetries and positions of intermolecular contacts (14-16). We believe that our analysis removes many of the ambiguities, especially with respect to the relative positions of intermolecular contacts in protofibrils and fibrin fibers. Based on the electron microscope images, and also on considerations of symmetry that have not been explicitly drawn in previous analyses, we conclude that there are three types of intermolecular contacts in fibrin. Two of these contacts are involved exclusively in the formation of the protofibril and are visualized in our images. The third type of contact is involved in the second step of polymerization, the lateral association of protofibrils.

\section{MATERIALS AND METHODS}

Human fibrinogen (Grade L, Kabi, Stockholm) was dissolved in $0.3 \mathrm{M} \mathrm{NaCl}$ and dialyzed at $4^{\circ} \mathrm{C}$ for $18 \mathrm{hr}$ in order to remove

† Present address: Dept. of Cell Biology and Anatomy, Johns Hopkins

University School of Medicine, Baltimore, MD 21205.

$\$$ To whom reprint requests should be addressed. 
free calcium. The solution was centrifuged at $30,000 \times \mathrm{g}$ for 20 min, divided into aliquots, and stored at $-70^{\circ} \mathrm{C}$.

Fibrinogen dimers were made as described (17) by crosslinking fibrinogen with factor XIIIa and purifying the dimers by chromatography on Sepharose 4B. Fibrin monomer was prepared by dissolving noncrosslinked fibrin in either $1 \mathrm{M}$ sodium bromide (18) or $0.1 \mathrm{M}$ acetic acid (19).

Intermediates in fibrin polymerization were prepared as described (13). Briefly, fibrinogen was diluted to $0.01 \mathrm{mg} / \mathrm{ml}$ in $0.05 \mathrm{M}$ Tris (pH 7.4) containing either 0.10 or $0.50 \mathrm{M} \mathrm{NaCl}$ and occasionally $0.005 \mathrm{M} \mathrm{CaCl}_{2}$. Negatively stained specimens were prepared at intervals after the addition of an excess (non-ratelimiting concentration) of thrombin.

\section{RESULTS}

Trimer Complexes. Fibrin monomer dissolved in $1 \mathrm{M}$ sodium bromide (18) or in $0.1 \mathrm{M}$ acetic acid (19) polymerized when diluted into buffer; however, polymerization was completely inhibited when the buffer contained a 7-fold molar excess of "end-to-end fibrinogen dimers." These fibrinogen dimers, whose preparation we have described elsewhere (17), consist of two fibrinogen molecules covalently crosslinked at their endto-end contact site and, thus, are equivalent to a two-molecule segment of a strand. Electron microscopy of negatively stained samples from a mixture containing a 3-fold excess of fibrinogen dimers over fibrin monomers (Fig. 1) shows a variety of structures. The most common structure, aside from trinodular fibrin monomers and crosslinked fibrinogen dimers, is a trimer complex consisting of a fibrin monomer molecule attached to a crosslinked fibrinogen dimer. In the complex, the central nodule of the thrombin-activated fibrin monomer is attached to the junction of the two outer nodules of the crosslinked fibrinogen dimer (Fig. 1 Right). In contrast, the two outer nodules of the fibrin monomer apparently do not interact with the central nodules in the fibrinogen dimer and, in fact, are often bent away. This is not surprising because the sites activated by thrombin are located in the central nodule, and the fibrinogen dimer has not been activated by thrombin.

Protofibrils. We identified protofibrils and examined their structure in specimens prepared at various times after the addition of thrombin to fibrinogen. The polymerization mixture sometimes included $0.5 \mathrm{M} \mathrm{NaCl}$ because high ionic strength prolongs the rate of gelation relative to the formation of protofibrils, although identical structures were seen also at physiological ionic strength. Fig. 2 shows negatively stained protofibrils found $15 \mathrm{~min}$ after thrombin addition. In favorable orientation, the protofibril appears to consist of two parallel lines, each line being a sequence of prominent, elongated "dashes" $7 \mathrm{~nm}$ thick by $15 \mathrm{~nm}$ long, which are separated by a $30-\mathrm{nm}$ space of poorly contrasted or less-dense material. Trinodular molecules in the protofibril are not distinguishable as

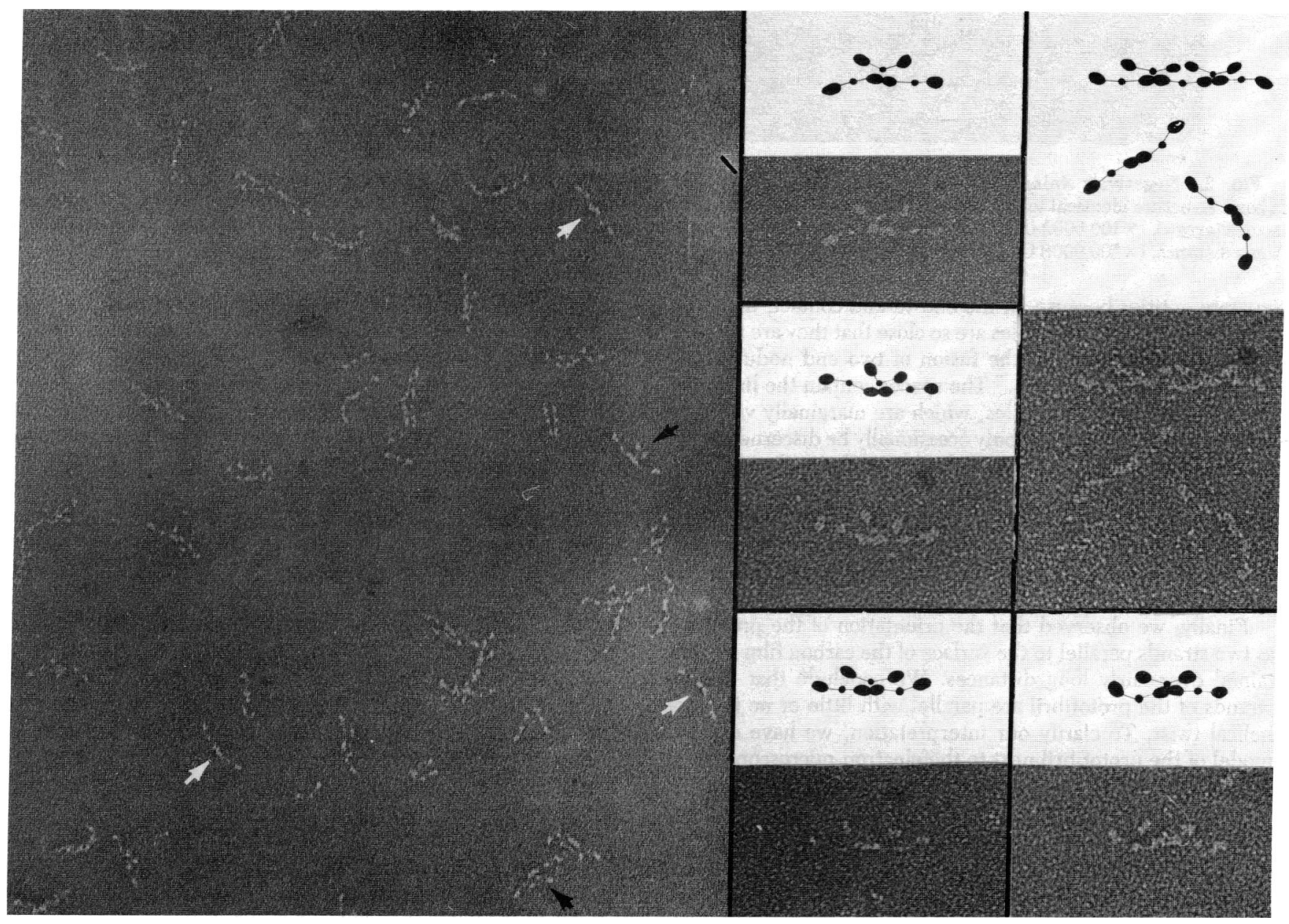

FIG. 1. Trimer complexes formed by diluting solubilized fibrin monomer into a solution of end-to-end fibrinogen dimers, which were covalently crosslinked by factor XIIIa (17). (Left) A variety of structures that are found in such a preparation, including trimers (black arrows), crosslinked fibrinogen dimers (white arrows), and unassociated trinodular fibrin monomer molecules. $(\times 100,000$.) (Right) Selected trimer complexes (and one pentamer complex) at higher magnification with interpretative drawings. $(\times 200,000$.) 


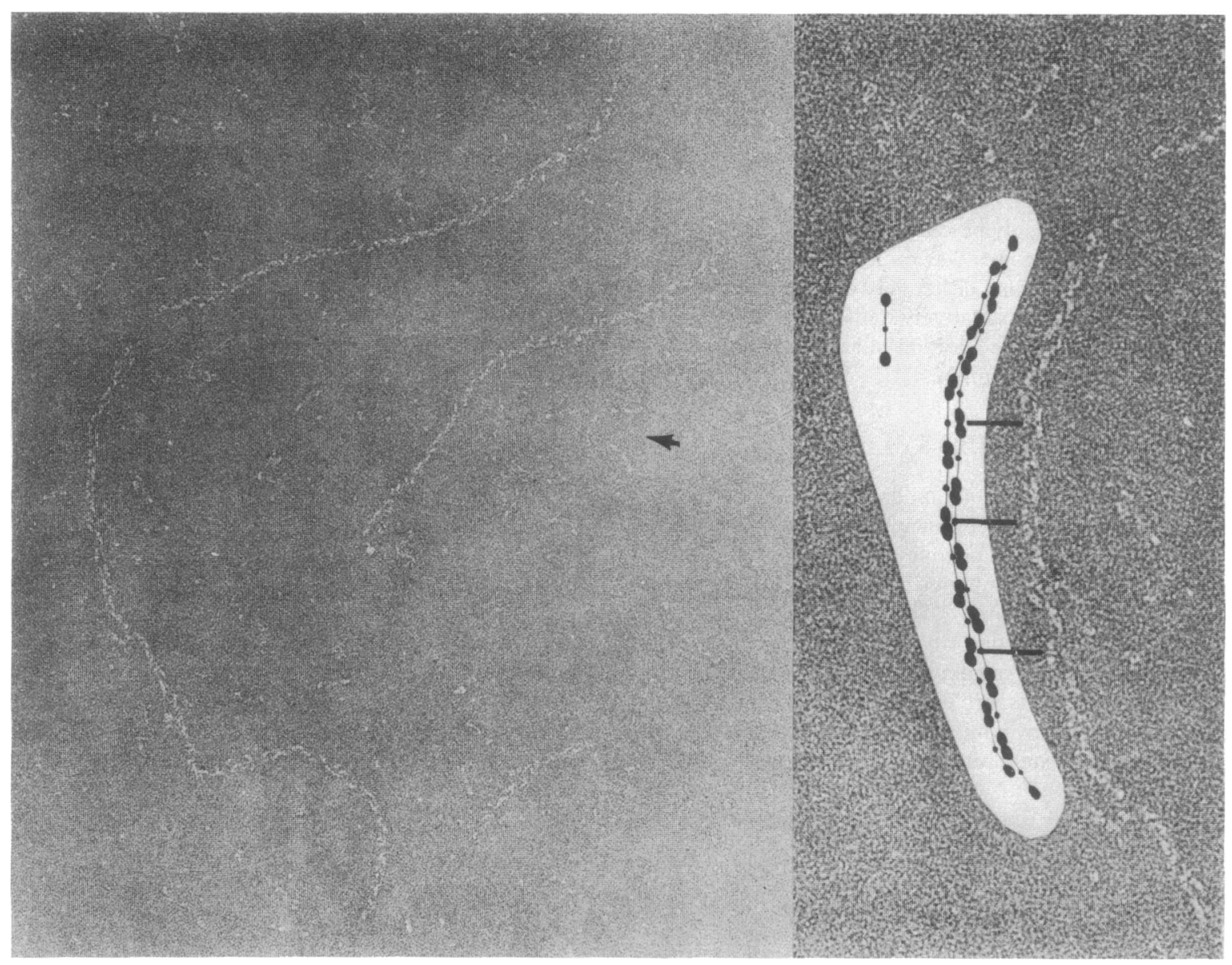

Fig. 2. Negatively stained fibrin protofibrils obtained after $15 \mathrm{~min}$ of polymerization in $0.5 \mathrm{M} \mathrm{NaCl}$ (13). In favorable orientation, they show a basic structure identical to that seen in the trimer complexes. (Left) Several protofibrils in random orientation and unassociated trinodular molecules (arrow). $(\times 100,000$.) (Right) A selected region of one of the protofibrils in which the fused D nodules of the two strands can be followed for some distance. $(\times 200,000$.) Compare with the interpretive drawing.

separate entities because, at the end-to-end contact, the outer nodules of adjacent molecules are so close that they are not separately resolved. Rather, the fusion of two end nodules produces the prominent "dash." The spaces contain the linker regions and the central nodules, which are marginally visible in isolated molecules and can only occasionally be discerned in the protofibril after the position of the trinodular molecule is established. This interpretation of the structure is substantiated by the images of crosslinked fibrinogen dimers (17) and the trimer complexes (Fig. 1), in which the fused end nodules are seen to have the same appearance and dimensions as the dashes in the protofibril.

Finally, we observed that the orientation of the protofibril as two strands parallel to the surface of the carbon film is maintained over fairly long distances. We conclude that the two strands of the protofibril are parallel with little or no intrinsic helical twist. To clarify our interpretation, we have drawn a model of the protofibril next to the electron-microscope image (Fig. 2 Right).

Interpretation-A Model of the Protofibril Based on Electron Micrographs and Considerations of Symmetry. In models of the structure and assembly of fibrin, the fibrin monomers are often represented with faceless spherical nodules. Even though the electron micrographs do not resolve the structure or "face" of the nodules, it is important to stress that each nodule is, in fact, a complex structure with specific sites or areas on its surface that will form the intermolecular contacts. We now can specify the location of some of these sites relative to a molecular frame of reference determined by the shape and symmetry of the fibrinogen molecule; furthermore, we can specify the relative positioning of fibrinogen molecules in dimers and trimers; finally, we can determine positioning of fibrin monomers relative to the frame of reference determined by the symmetry of the protofibril. We first shall summarize the model and then discuss why we believe it to be correct.

The contacts and orientation of the molecules in the model of the protofibril are identified in Fig. $3 a$. The important features are that $(i)$ all the molecules in one strand face in the same direction, (ii) the two strands face each other, and (iii) the two strands are staggered by one-half the molecular length. The structural features may be described more concisely by saying that the molecules in the protofibril are related (at least approximately) by a two-fold screw axis between the two strands. The intramolecular two-fold axes may be said to define the plane of the protofibril. A second set of two-fold axes runs perpendicular to this plane and intersects the protofibril axis midway between the first set.

The association of molecules into strands involves a contact between the distal ends of the outer nodules. We shall call this the DD-long contact to indicate that it is a longitudinal association between the $\mathrm{D}$ nodules. [In this discussion, we shall refer to the outer and central nodules as the $D$ and $E$ nodules, respectively $(6,7)$.] This contact has been identified as the site of the $\gamma$-chain crosslink (17) and is the only contact present in the crosslinked fibrinogen dimer.

The most important bond in the protofibril is the contact 


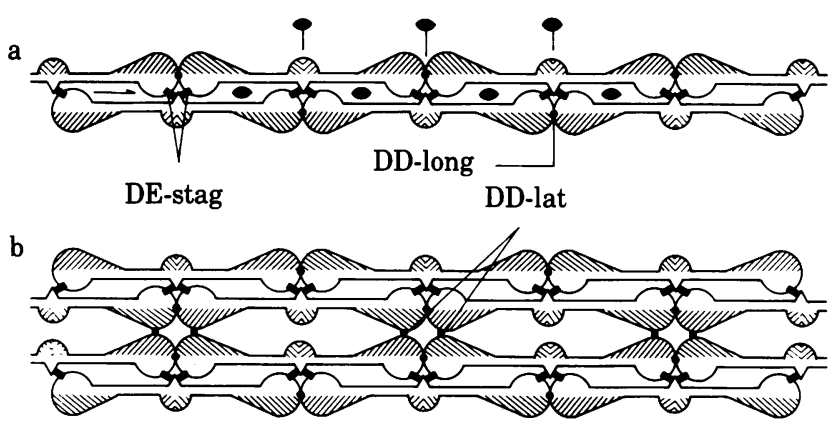

c

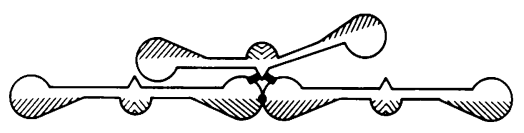

FiG. 3. (a) Model of the two-stranded protofibril with the two-fold screw axis between strands and the intra- and intermolecular two-fold axes. The two contacts involved in protofibril formation are identified as DD-long and DE-stag. The sites involved in these contacts are completely saturated within the protofibril, except for the two ends. (b) Lateral association of protofibrils to make the fibrin fiber. This association must involve a different type of lateral contact, probably between D nodules, identified as DD-lat. The two protofilaments are not coplanar, as suggested by this diagram, but are arranged around 3- or 4-fold axes to form a three-dimensional lattice (14). It is also important to realize that there are two DD-lat bonds per $\mathrm{D}$ nodule (see text). (c) Model of fibrin-fibrinogen trimer. Our earlier schematic drawing in ref. 17 showed the fibrinogen dimer rotated $90^{\circ}$ about its axis from the correct position. This orientation was required to show the structure of the crosslink, but it is inconsistent with the symmetry of the protofibril, which is shown explicitly here.

between the central nodule of one molecule and the junction of two outer nodules in the other strand. This contact, specifically the interface on the $\mathrm{E}$ nodule, is apparently at or near the site where fibrinopeptide $A$ is removed by thrombin. We shall call this "the DE-stag contact" to indicate that it is a staggered lateral contact between a D nodule of one strand and an E nodule in the other. There are actually two identical DE-stag contacts per molecule because the E nodule consists of two identical halves, each of which contacts one D nodule in the adjacent strand.

The proposed symmetry of the protofibril means that all of the DE-stag contacts are complete within a single protofibril (i.e., all of these contact surfaces face inwards and are interfaced to complementary surfaces on the adjacent strand). This means that the protofibril is "closed" with respect to lateral growth, and this particular contact cannot be used for addition of a third strand to the protofibril. One should note that the resolution of the electron micrographs is not sufficient to show the polarity of the nodules directly. The proposed symmetry is certainly suggested by the images, but the strongest evidence for it is the observation from both light scattering and electron microscopy that the two-stranded protofibril exists as a discrete species.

The significance of this model for the contacts and molecular arrangement in fibrin is perhaps best illustrated by comparison with a different type of model presented by Olexa and Budzynski (15). In this model (Fig. 4), all of the strands face the same direction. In this case, the two-stranded protofibril would not be a distinct structural intermediate because a third and succeeding strands could be added, using the same DE-stag contacts, which are always exposed on the free edge. This type of "open" polymerization is not easily compatible with the evidence showing the two-stranded protofibrils to be the predominant polymer species at early times of assembly.

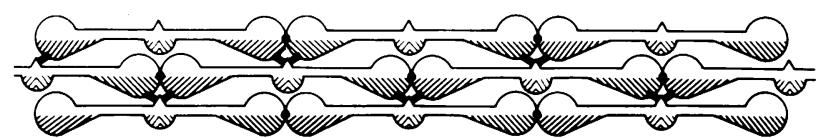

Fig. 4. A different model for lateral packing in which the strands all face the same way. In this case, a single type of staggered lateral contact would generate sheets of two, three, or more strands. This model is inconsistent with the identification of the two-stranded protofibril as a distinct structural intermediate.

The second of the two steps in fibrin polymerization is the association of protofibrils to form thicker fibers $(9,10,13)$. The thrombin-activated DE-stag sites are all saturated within the protofibril; therefore, this association must involve a different class of lateral contacts. Because the D nodules are larger and protrude from the protofibril more than the E nodules, we suggest that these lateral contacts are between $D$ nodules in adjacent protofibrils; this further implies that the strands involved in these contacts are in register (see also ref. 14). We shall refer to these as the DD-lat contacts (Fig. $3 b$ ). There must be two DD-lat contacts per $\mathrm{D}$ nodule, pointing in different directions, in order to form a three-dimensional fiber rather than a sheet. Information about the lateral packing is still too tentative to specify the symmetry of these bonds or the unit cell.

\section{DISCUSSION}

The electron micrographs show the location and orientation of the DE-stag and DD-long contacts. The nature of the DD-lat contact is more speculative because there is very little structural evidence for the arrangement of protofibrils within the fibrin fiber or for the position of the contacts between them. The possible arrangements may be greatly restricted, however, if one assumes that the structure is crystallographic (i.e., that all molecules have identical positions and contacts; ref. 14) and if the symmetry of the protofibril is specified. This approach is considerably strengthened by the recent work of Torbet et al. (20), which shows that the lateral packing in oriented fibrin gels is crystalline. Their diffraction pattern is consistent with two possible space groups, each of which could be built from protofibrils with the two-fold screw symmetry we have deduced. There is still some problem, however, in constructing a model with the low density calculated for the fibrin fiber (14).

It has been observed that the kinetics of polymerization, in particular the lateral association of protofibrils, are much slower if the fibrinogen is activated by reptilase, which cleaves fibrinopeptide A but releases fibrinopeptide B very slowly if at all (10, $21)$. This and related observations have led to the suggestion that release of fibrinopeptide $B$ exposes a new contact site involved in the lateral association of protofibrils $(15,21)$. We have found that, although the rate of polymerization is much slower for reptilase-activated fibrinogen, the structures seen by electron microscopy (protofibrils, small and large banded fibers) are essentially the same as those seen at corresponding times in the polymerization of thrombin-activated fibrinogen (13). Because cleavage of fibrinopeptide $A$ is sufficient to produce all of the structures seen in polymerization with thrombin, we do not feel that a separate contact site involving fibrinopeptide B is required in the model.

The possibility of more than one type of lateral contact between protofibrils cannot be excluded, but the hypothesis that there is only a single class of DD-lat contacts is simple and attractive. We should note, in particular, that the existence of branch points does not require a separate type of lateral contact but would be a natural outcome of the association of long flexible protofibrils $(9,10,13)$. The bend required at the branch point could be placed within the fibrin monomers, which are known 
to be flexible, with the intermolecular contacts remaining relatively rigid. It is no doubt important that the fibrin lattice is very loose (22), with large spaces or holes between molecules that permit the branching fibers to interdigitate.

The DD-long and DD-lat contacts, which are spatially removed from the $\mathrm{E}$ nodule, are presumably not affected by thrombin activation. These contacts and the unactivated DEstag sites must be relatively weak intersubunit bonds because there is no significant association of unactivated fibrinogen molecules. The activated DE-stag contact, on the other hand, must be a reasonably strong subunit association because the trimer complexes remain stable at $3 \mu \mathrm{g} / \mathrm{ml}$ of protein. This attachment comprises two of the DE-stag contacts, each of the activated sites on the fibrin $\mathrm{E}$ nodule contacting one of the crosslinked $\mathrm{D}$ nodules of the dimer. The cooperative association forming two bonds is expected to be orders of magnitude stronger than formation of a single bond (23). Thus, a fibrin monomer attached to a terminal D nodule by one DE-stag contact is never seen (Fig. 1).

In the generally accepted scheme for fibrin assembly, the first polymerization product formed after activation by thrombin is a staggered overlap dimer, which is the smallest fragment of a protofibril. This dimer also has two DE-stag contacts, one from each $\mathrm{E}$ nodule to a $\mathrm{D}$ nodule on the other molecule. Therefore, it should be as stable as the trimer complex. Smith's claim (16) of having isolated extremely stable dimers, in which only one of the DE-stag sites was activated on each $E$ nodule, is consistent with this expectation. The formation of dimers and the growth of larger protofibrils is a virtually irreversible association, driven by the strong bond energy of the DE-stag contact. The relatively weak DD-long contact probably does not play an important role in this polymerization; this again agrees with the observation of a lack of cooperativity in kinetics of formation of protofibrils from fibrin molecules (10).

The second stage in fibrin polymerization, the lateral association of protofibrils, is effected by the formation of the DDlat contact. The bond strength of this contact is apparently quite weak because the contact fails to produce a significant association of individual molecules. In the association of preformed protofibrils, however, a number of these bonds will be made at once in a cooperative fashion. This association should become much stronger as the length of the protofibril and, thus, the number of DD-lat bonds increases (see ref. 23 for a more detailed treatment of this point). In this model, the lateral association of protofibrils does not directly involve thrombin activation but occurs spontaneously as soon as the protofibrils have grown past a critical length, in agreement with experiment $(10$, 13).

In summary, our work has provided a more detailed structural model for the fibrin protofibril and has led to new insights on the mechanism of polymerization. The structural model is based on electron micrographs of negatively stained protofibrils and closely related trimer complexes, which show the molecular arrangement more clearly than previous studies. Of particular importance, the protofibril is seen to have two-fold; rather than three- or four-fold symmetry-a conclusion that greatly restricts the possible packing models for fibrin (14). Our observations and interpretation support the two-step mechanism of fibrin polymerization and demonstrate the two types of intermolecular contacts within the protofibril. In addition, the model that we developed strongly suggests the existence of a third type of contact between protofibrils.

We thank Dr. William Longley for helpful discussions. We also thank S. Olexa for pointing out that our previous diagram is inconsistent with the symmetry of the protofibril that we propose in the present manuscript. The work was supported by National Institutes of Health Research Grants HL-23454 (H.P.E.) and HL-20319 (J.H.).

1. Hall, C. \& Slayter, H. (1959) J. Biophys. Biochem. Cytol 5, $11-15$.

2. Fowler, W. E. \& Erickson, H. P. (1979) J. Mol Biol. 134, 241-249.

3. Weisel, J. W., Phillips, G. M. \& Cohen, C. (1981) Nature (London) 289, 263-267.

4. Tooney, N. \& Cohen, C. (1977) J. Mol. Biol. 110, 363-385

5. Doolittle, R. F. (1977) Horizons Biochem. Biophys. 3, 164-191.

6. Fowler, W. E., Fretto, L. J., Erickson, 'H. P. \& McKee, P. A. (1980) J. Clin. Invest. 66, 50-56.

7. Telford, J. N., Nagy, J. A., Hatcher, P. A. \& Scheraga, H. A. (1980) Proc. Nath. Acad. Sci. USA 77, 2372-2376.

8. Stryer, L., Cohen, C. \& Langridge, R. (1963). Nature (London) 197, 793-794.

9. Ferry, J. D. (1952) Proc. Natl. Acad. Sci. USA 38, 566-569.

10. Hantgan, R. \& Hermans, J. (1979) J. Biol. Chem. 254, 11272-11281.

11. Krakow, W., Endres, G., Siegel, B. \& Scheraga, H. (1972) J. Mol. Biol. 71, 95-103.

12. Slayter, H. (1976) Ultramicroscopy 1, 341-357.

13. Hantgan, R., Fowler, W., Erickson, H. \& Hermans, J. (1980) Thromb. Haemosts. (Stuttgart) 154, 367-375.

14. Hermans, J. (1979) Proc. Natl. Acad. Sci. USA 76, 1189-1193.

15. Olexa, S. \& Budzynski, A. (1980) Proc. Nath Acad. Sci. USA 77, 1374-1378.

16. Smith, G. (1980) Biochem. J. 185, 1-11.

17. Fowler, W. E., Erickson, H. P., Hantgan, R., McDonagh, J. \& Hermans, J. (1981) Science 211, 287-289.

18. Sturtevant, J., Laskowski, M., Donnelley, T. \& Scheraga, H. (1955) J. Am. Chem. Soc. 77, 6158-6172.

19. Belister, V., Varetskaja, T. \& Maineva, G. (1968) Biochim. Biophys. Acta 154, 367-375.

20. Torbet, J., Freyssinet, J. \& Hudry-Clergeon, G. (1981) Nature (London) 289, 91-93.

21. Blombäck, B., Hessel, B., Hogg, D. \& Therkildsen, L. (1978) Nature (London) 275, 501-505.

22. Carr, M. E. \& Hermans, J. (1978) Macromolecules 11, 46-50.

23. Erickson, H. P. \& Pantaloni, D. (1981) Biophys. J., 34, 293-309. 